\title{
Reimplante de falange distal de dedo sin anastomosis venosa con anestesia local
}

\author{
Federico Paganini, Mariano Alberghini, Natalia Gutiérrez Olivera, Christian A. Allende Nores \\ Servicio de Cirugía de la Mano y Cirugía Reconstructiva de los Miembros, Sanatorio Allende, Córdoba, Argentina
}

\begin{abstract}
RESUMEN
Presentamos a un paciente que sufrió una amputación traumática de la falange distal del $5^{\text {to }}$ dedo de su mano izquierda, y fue tratado mediante un reimplante sin anastomosis venosa. En el mismo acto quirúrgico, se le realizó una artrodesis definitiva con clavijas. La cirugía se efectuó con anestesia troncular del dedo, por lo cual no se requirió de un anestesista ni fue necesario un tiempo adecuado de ayuno. Debido a la localización distal de la amputación y a la ausencia de una vena dorsal viable para ser anastomosada, se optó por un drenaje venoso controlado mediante la extracción de la placa ungueal, el frotado del lecho ungueal y la colocación periódica (cada $3 \mathrm{~h}$, por 7 días) de una gasa impregnada con heparina. Además, el paciente fue anticoagulado con enoxaparina $40 \mathrm{mg}$ y ácido acetilsalicílico cada $24 \mathrm{~h}$, por 21 días. El reimplante fue exitoso.
\end{abstract}

Palabras clave: Reimplante de dedo; sin anastomosis venosa; anestesia local.

Nivel de Evidencia: IV

\section{Distal phalanx replantation under local anesthesia and without venous anastomosis}

\begin{abstract}
We present a patient who experienced a traumatic amputation of his left fifth finger distal phalanx, and was treated using replantation without venous anastomosis. The same surgical procedure also included a final arthrodesis with Kirschner wires. Surgery was performed with digital block anesthesia, which did not require the presence of an anesthesiologist nor fasting. The distal level of the amputation and the lack of viable options for dorsal vein anastomosis motivated our decision for venous drainage controlled by nail-plate removal, nail-bed scrubbing and periodic (every 3 hours during 7 days) dressing with heparin-impregnated gauze. In addition, the patient received anticoagulant therapy with aspirin and enoxaparin $40 \mathrm{mg}$ every 24 hours for 21 days. The replantation procedure was a success.
\end{abstract}

Key words: Finger replantation; no venous anastomosis; local anesthesia.

Level of Evidence: IV

\section{INTRODUCCIÓN}

Los avances en las técnicas microquirúrgicas y en el instrumental de microcirugía, así como el mayor entrenamiento de los cirujanos de mano, han hecho posible que los reimplantes de los extremos distales de los dedos puedan realizarse con una tasa de éxito del $70 \%$ al $90 \%$ cuando una arteria y una vena son reparadas. ${ }^{1}$

Sin embargo, un reimplante a dicho nivel no deja de representar un desafío aun para el más experimentado de los cirujanos, pues requiere de una técnica microquirúrgica precisa. Es bien sabido que, al realizar un reimplante de dedo, se busca restablecer no solo la función de la mano, sino también su imagen estética. Restaurar el largo del dedo tiene la misma importancia que restaurar su matriz y su lecho ungueal, ya que le ofrece al paciente un bienestar no sólo psicológico, sino también estético. ${ }^{2,3}$ Por otro lado, también es igual de importante la reparación de los nervios colaterales digitales, con el fin de evitar futuros neuromas y recuperar la sensibilidad.

Las amputaciones a nivel de la articulación interfalángica distal o distales a ella plantean una gran dificultad para realizar las anastomosis venosas debido a la inadecuada calidad (secundaria al trauma) o incluso a la ausencia de estas. ${ }^{4.5}$ Cuando no se puede hacer dicha anastomosis, es necesario buscar vías alternativas que permitan un

Recibido el 14-4-2019. Aceptado luego de la evaluación el 20-6-2019 • Dr. FEDERICO PAGANINI • federicopaganini@ hotmail.com (ID https://orcid.org/0000-0002-1162-0956 Cómo citar este artículo: Paganini F, Alberghini M, Gutiérrez Olivera N, Allende Nores CA. Reimplante de falange distal de dedo sin anastomosis venosa con anestesia local. Rev Asoc Argent Ortop Traumatol 2020;85(3):246-253. https://doi.org/10.15417/issn.1852-7434.2020.85.3.981 
drenaje venoso continuo y un flujo sanguíneo constante en el segmento reimplantado hasta que el drenaje venoso se restablezca naturalmente mediante neovascularización. Por este motivo, existen diferentes técnicas alternativas a la anastomosis venosa, con distintas tasas de éxito, y el único propósito de descongestionar el segmento reimplantado. Las técnicas utilizadas con más frecuencia se basan en la hemorragia o el sangrado externo y, entre ellas, podemos mencionar el uso de sanguijuelas medicinales, ${ }^{6}$ el sangrado controlado del lecho ungueal ${ }^{7}$ y el sangrado controlado a través de incisiones paraungueales con la colocación de heparina sódica ${ }^{8}$. Además, hay técnicas que no implican el sangrado externo, como las fístulas arteriovenosas ${ }^{9,10}$ y venocutáneas ${ }^{11}$. En nuestro caso, aplicamos un protocolo de drenaje venoso similar al de Erken y cols., ${ }^{7}$ que consiste en aplicar y frotar una gasa impregnada con heparina sódica en el lecho ungueal después de extraer la placa ungueal durante la cirugía.

Todas estas técnicas quirúrgicas siempre se asocian a protocolos de anticoagulación, basados en heparina sódica y antiagregación plaquetaria.

A continuación, presentamos un caso de amputación de un segmento distal de un dedo que fue reimplantado con anestesia local y sin anastomosis venosa, la cual fue reemplazada por un sangrado continuo controlado asociado a un plan sistémico de anticoagulación.

\section{CASO CLÍNICO}

Hombre de 26 años, diestro, que sufre una amputación traumática a nivel de la articulación interfalángica distal del $5^{\text {to }}$ dedo de la mano izquierda al manipular una sierra circular. Según las clasificaciones de Tamai ${ }^{12}$ y de Ishikawa, ${ }^{13}$ el nivel de amputación era Tamai tipo II o Ishikawa subzona IV (Figura 1).

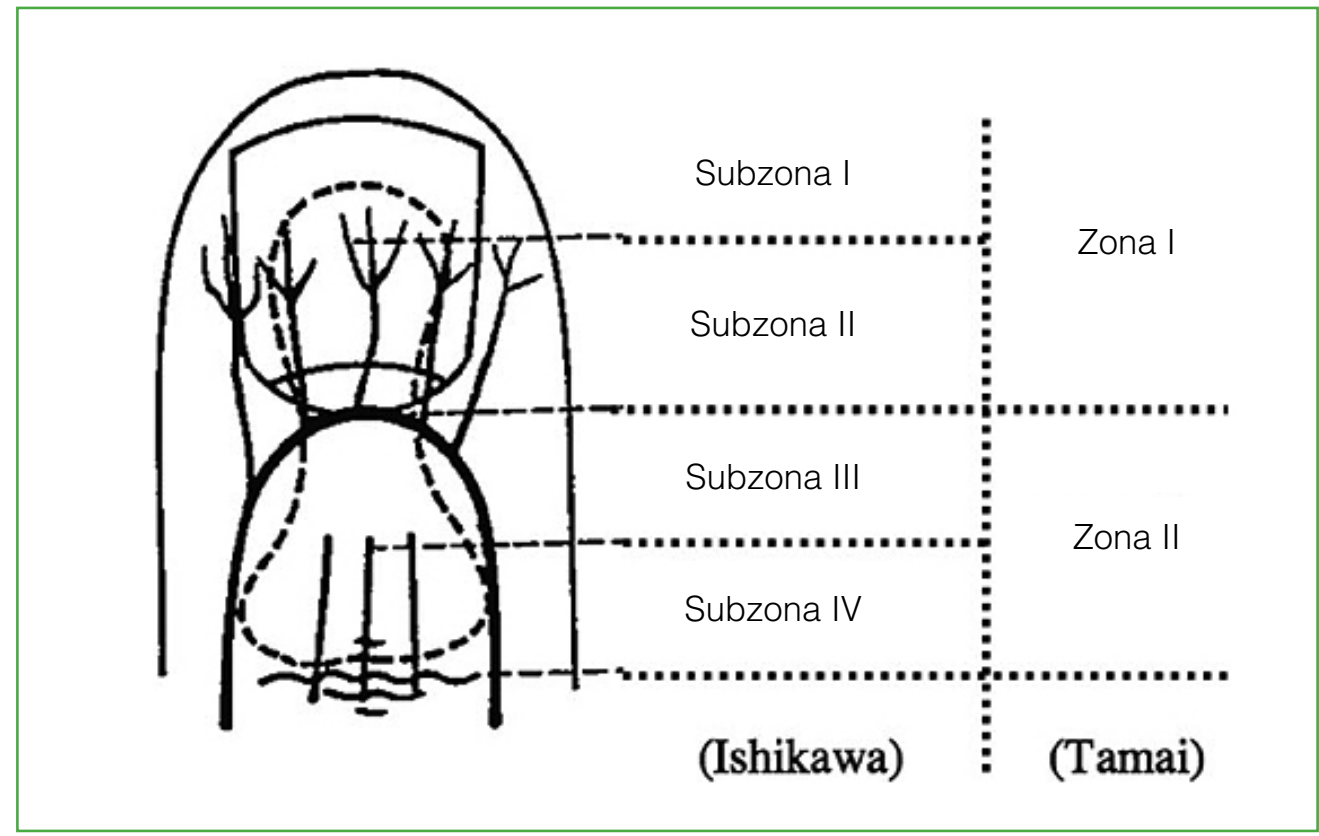

Figura 1. Clasificación de Ishikawa y Tamai.

El paciente concurrió, a las $2 \mathrm{~h}$ del accidente, con el extremo del dedo amputado (Figura 2) correctamente preservado en un recipiente con hielo y compresas húmedas. Su pasatiempo era tocar la guitarra, y resaltó la necesidad de conservar, en lo posible, la mayor longitud de dicho dedo. 


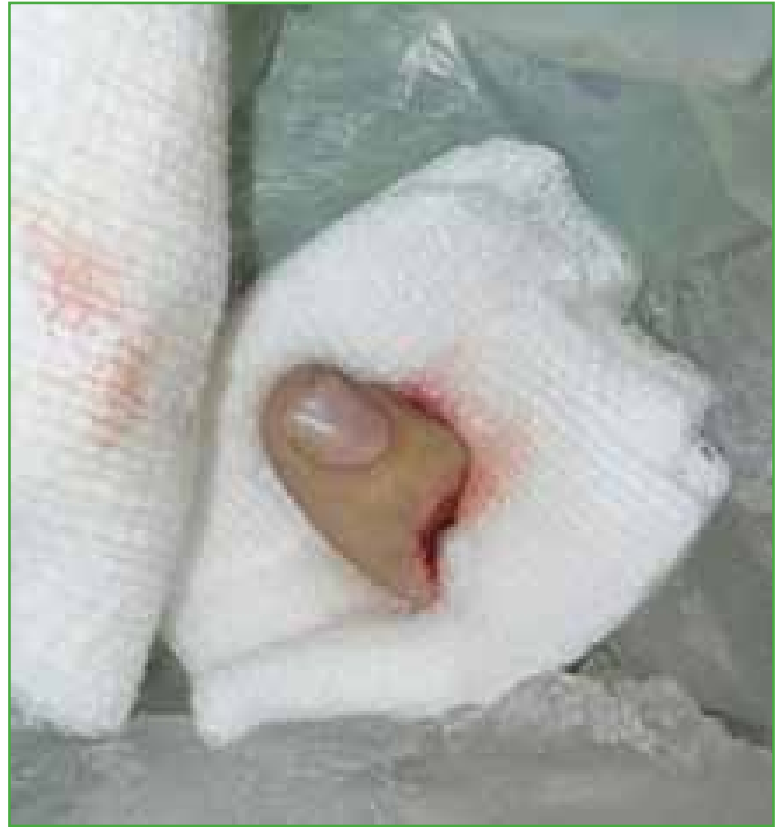

Figura 2. Segmento amputado, correctamente preservado en un recipiente con gasas húmedas y hielo.

Se decidió realizar el reimplante (Figura 3) inmediatamente en el quirófano. Se le administró anestesia troncular (local) del dedo, y se procedió a la limpieza y la artrodesis con dos agujas endomedulares de Kirschner de 1 mm (Figura 4). Posteriormente se realizó una tenorrafia flexora y extensora, y por último, se procedió a la sutura neurovascular con lupas microquirúrgicas de 3,5 de aumento. Tras la neurorrafia con Ethilon 9.0, se llevó a cabo exitosamente la anastomosis arterial con la misma sutura. Inmediatamente después de la arteriorrafia, se le administró al paciente una dosis intravenosa de heparina sódica de $70 \mathrm{U} / \mathrm{kg}$ de peso. Luego, se intentó una anastomosis venosa, pero no fue posible debido al daño presente y al pequeño calibre de las vénulas. En consecuencia, se optó por resecar la placa ungueal y colocar gasas impregnadas con heparina sódica de 5000 UI en el lecho ungueal, con recambio cada tres horas. No hicimos incisiones paraungueales de descarga. El tiempo total de cirugía fue de 150 minutos.

En el posoperatorio inmediato, se instruyó al paciente, a los familiares y a enfermería a que estimularan la zona de sangrado frotándola con una gasa embebida en heparina. Se le administró enoxaparina $40 \mathrm{mg} /$ día, por vía subcutánea y ácido acetilsalicílico $100 \mathrm{mg}$ /día, durante tres semanas (Figura 5). El paciente permaneció internado en una sala común calefaccionada a $24^{\circ} \mathrm{C}$ por el término de siete días. No tuvo complicaciones posoperatorias (no fue necesario realizar una transfusión sanguínea).

La artrodesis de la articulación interfalángica distal consolidó sin problemas y las clavijas se extrajeron a los dos meses (Figura 6). La artrodesis consolidó con $0^{\circ}$ de flexión y $10^{\circ}$ de pronación (el paciente se negó a una nueva cirugía correctiva). Tanto la articulación interfalángica proximal como la metacarpofalángica recuperaron la movilidad completa a los 60 días (Figura 7). 

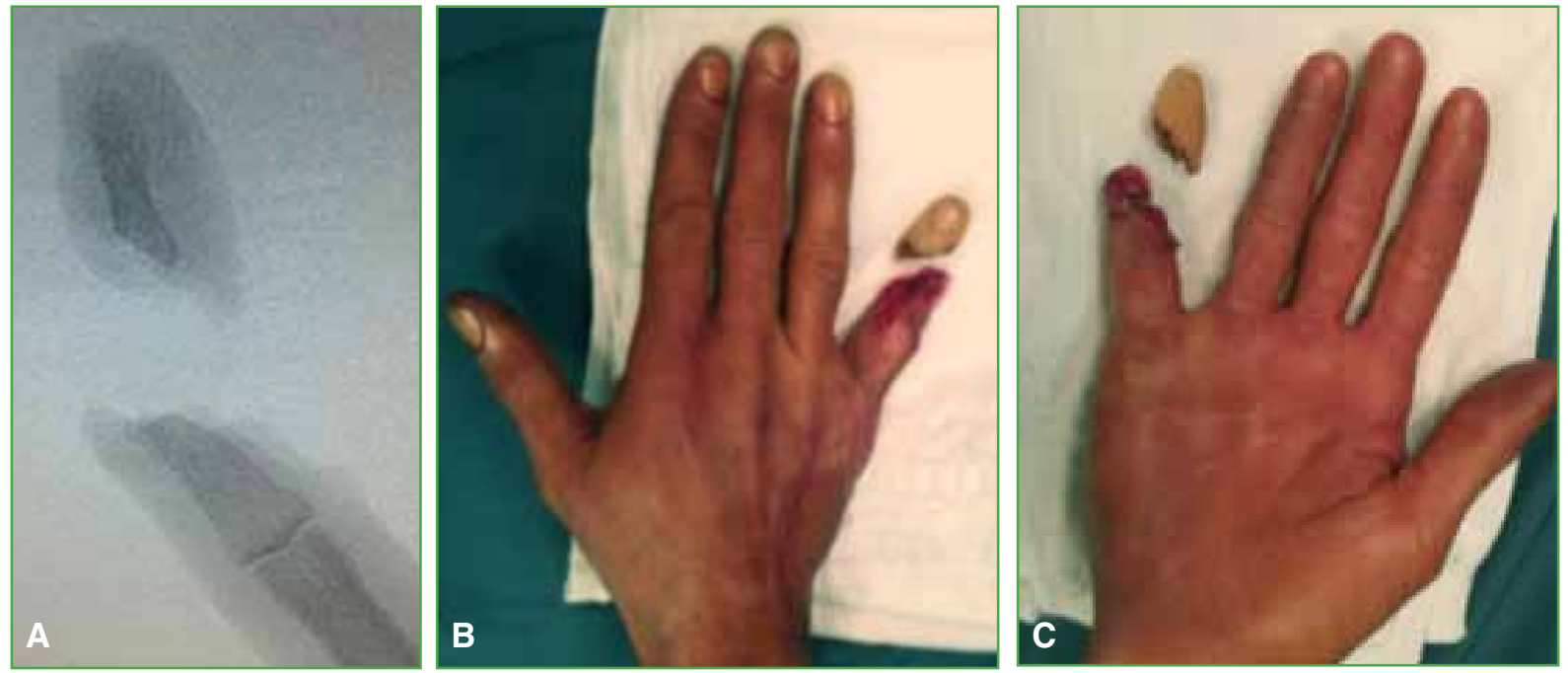

Figura 3. A. Radiografía intraoperatoria. Se observa el nivel óseo de amputación. B y C. Imágenes de la pieza por reimplantar.
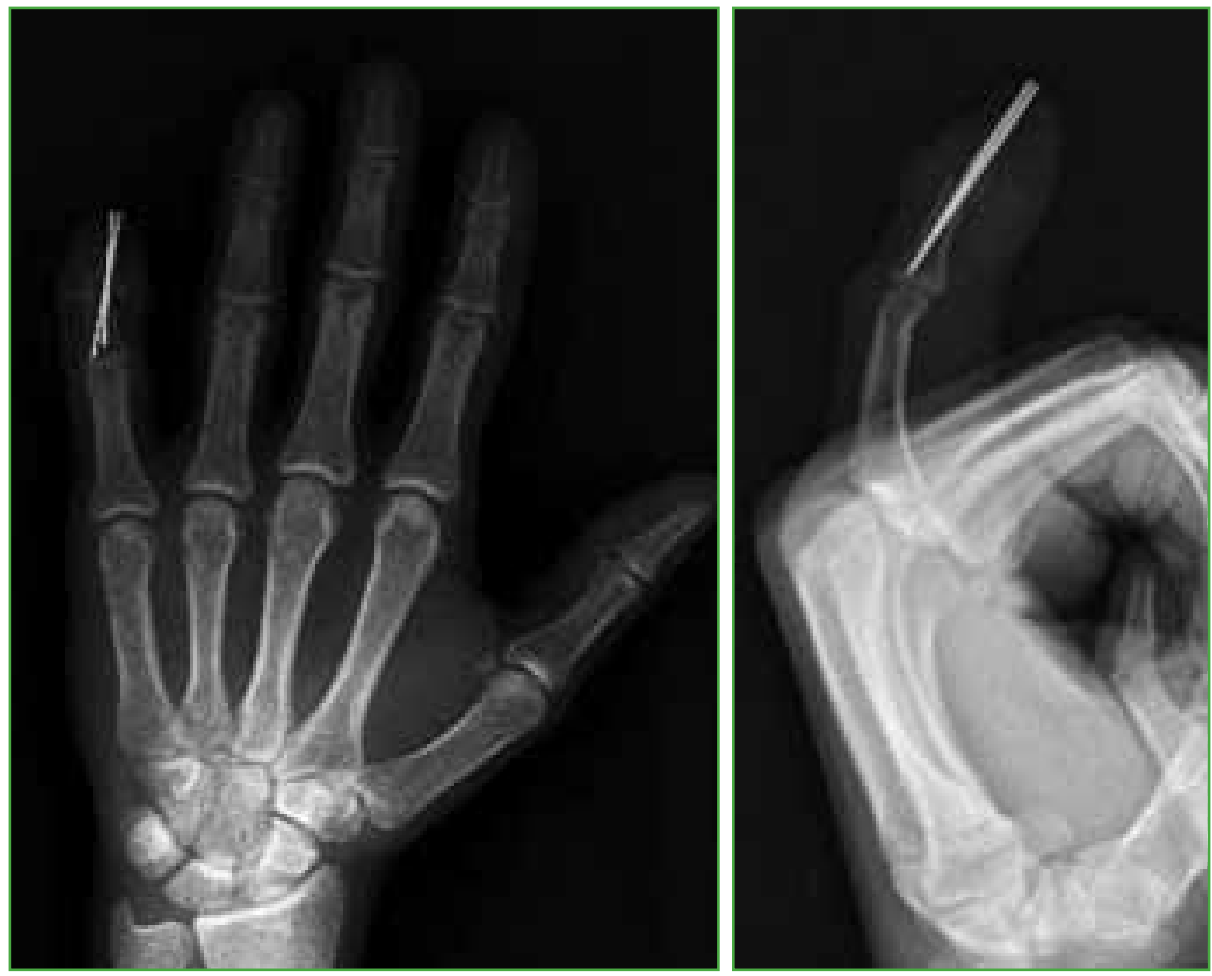

Figura 4. Artrodesis transitoria con dos clavijas endomedulares de Kirschner de $1 \mathrm{~mm}$. 


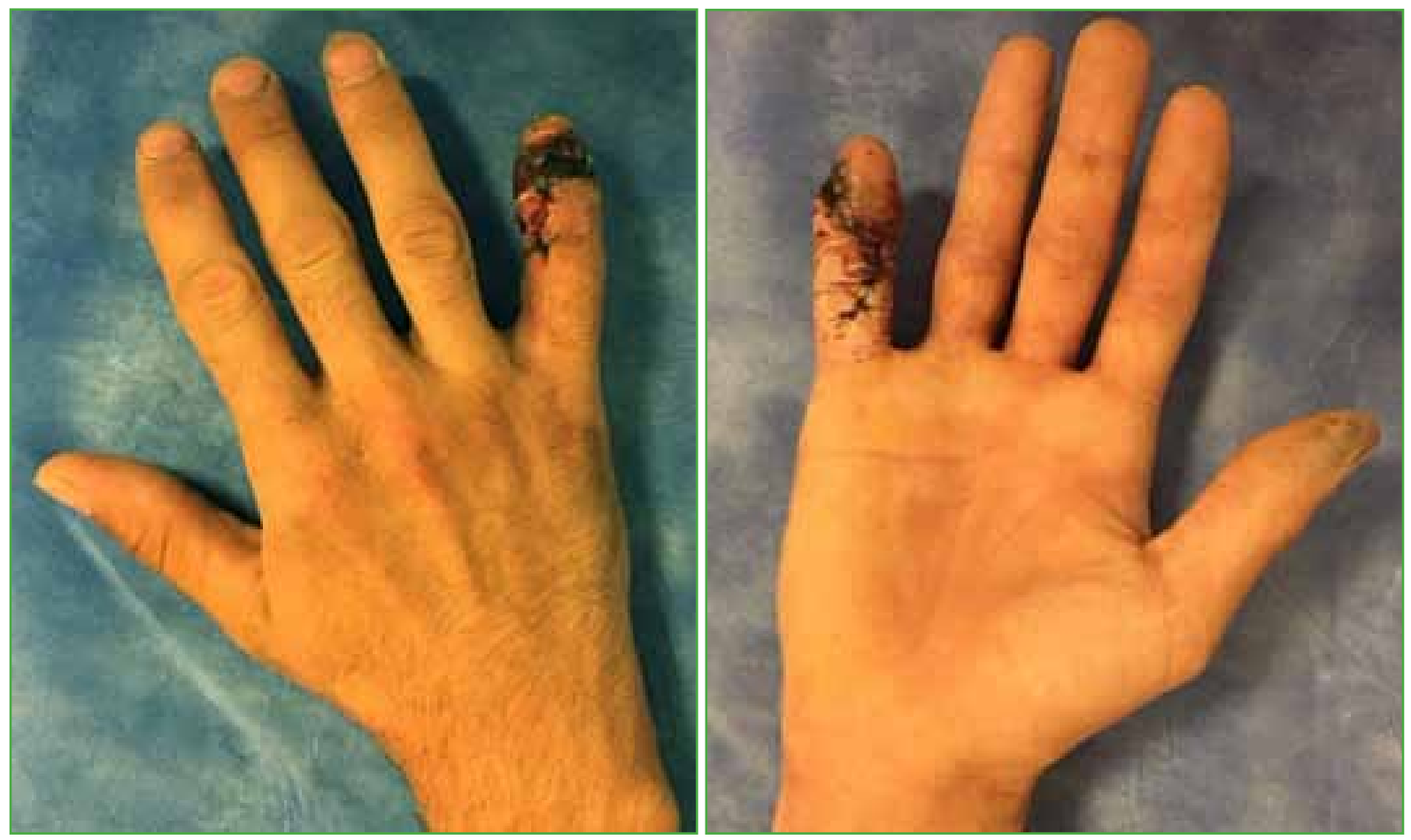

Figura 5. Reimplante del extremo distal del $5^{\text {to }}$ dedo, a las tres semanas de la cirugía.
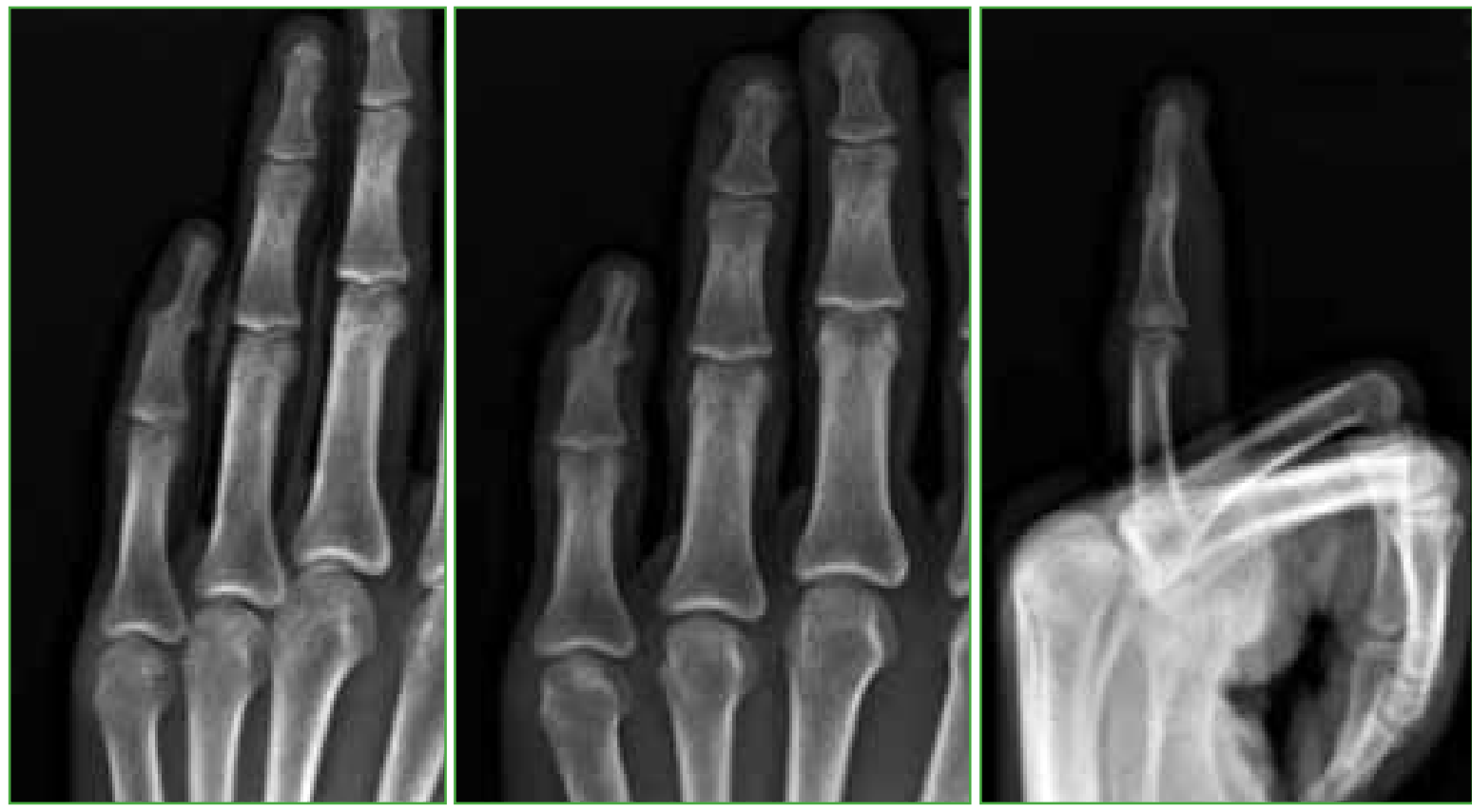

Figura 6. Radiografías a los dos meses de la cirugía. Se observa la consolidación de la artrodesis de la articulación interfalángica distal. 


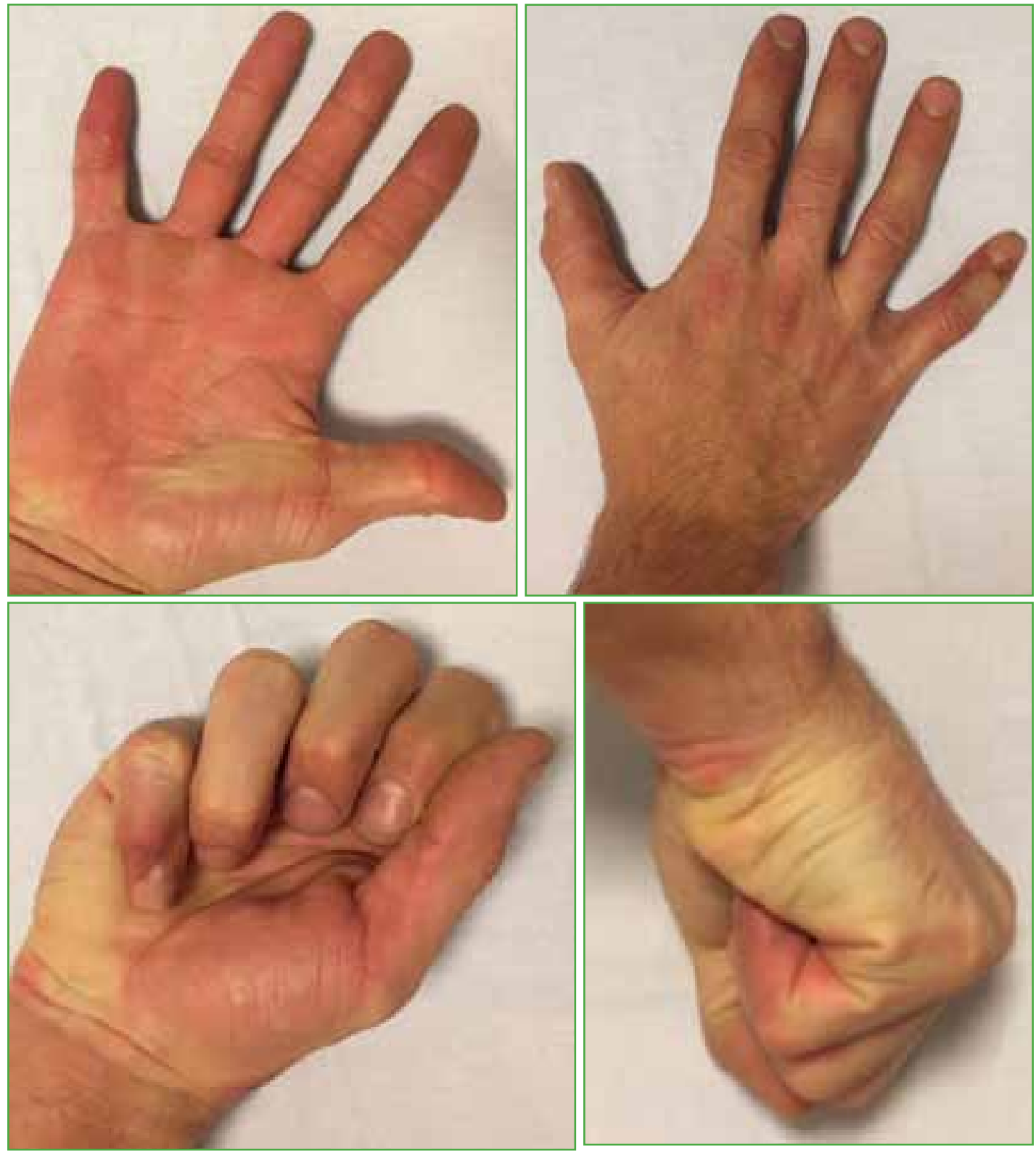

Figura 7. Resultado final. Recuperación completa de la movilidad de las articulaciones metacarpofalángica e interfalángica proximal, y la leve desviación en pronación del segmento reimplantado.

\section{DISCUSIÓN}

El reimplante de las amputaciones de las falanges distales de los dedos requiere de un adecuado entrenamiento médico en técnicas microquirúrgicas, y el éxito no sólo depende de la experiencia del microcirujano, sino también de factores extrínsecos, como el mecanismo de amputación, el tiempo de evolución y la adecuada conservación de la pieza amputada. Además, también intervienen factores intrínsecos del paciente, como la edad, si es tabaquista o no y la presencia de enfermedades que puedan comprometer la microcirculación periférica (diabetes). Por todo ello, Hui-Fu Huang y cols. ${ }^{14}$ afirman que el reimplante exitoso de un dedo a nivel de la articulación interfalángica distal no depende exclusivamente de la anastomosis venosa. 
En nuestro caso, utilizamos la técnica descrita por Erken y cols. ${ }^{7}$ que consiste en la anastomosis arterial, la colocación de gasas impregnadas con heparina en el lecho ungueal y un plan de anticoagulación con heparina y aspirina. Nuestro paciente permaneció internado siete días, en una sala común, al igual que en la serie de Erken y cols. (promedio 7 días), pero 15 de 24 pacientes requirieron una transfusión (promedio 1,2 U), mientras que nuestro paciente no la necesitó. Vale aclarar que estos autores afirman que la sensibilidad del segmento amputado a los dos años de la cirugía es similar independientemente de si se hace o no la neurorrafia digital; sin embargo, en nuestro caso, optamos por realizarla. Finalmente, obtuvieron una tasa de éxito del reimplante del $88 \%$.

En otra serie, Buntic y Brooks ${ }^{6}$ lograron un reimplante exitoso en todos sus casos. Efectuaron 19 reimplantes distales en 17 pacientes a quienes no se les realizó una anastomosis venosa y se les aplicó un protocolo de anticoagulación con dextrano 40, heparina intravenosa y sanguijuelas medicinales, la estancia hospitalaria fue más prolongada (promedio 9 días) y, además, 15 pacientes requirieron una transfusión de sangre.

Por último y, sobre la base de lo ya expuesto, podemos decir que, cuando no es posible realizar la sutura venosa en una amputación a nivel de la articulación interfalángica distal, se dispone de diferentes técnicas quirúrgicas que logran tasas de éxito más que aceptables.

\section{CONCLUSIONES}

Creemos que la técnica que utilizamos es válida, sencilla y reproducible, acorta los tiempos quirúrgicos de un reimplante, a la vez que prescinde de un anestesista. Sin embargo, requiere una internación más prolongada, que incrementa el costo y de un paciente y familiares responsables y capaces de cumplir el tratamiento. La principal limitación de esta técnica es que está reservada para las amputaciones a nivel de la articulación interfalángica distal o distales a ella.

Conflicto de intereses: Los autores no declaran conflictos de intereses.

ORCID de M. Alberghini: https://orcid.org/0000-0001-6793-0550

ORCID de N. Gutiérrez Olivera: https://orcid.org/0000-0002-3021-1277

ORCID de C. A. Allende Nores: https://orcid.org/0000-0002-2336-2157

\section{BIBLIOGRAFÍA}

1. Hattori Y, Doi K, Sakamoto S, Yamasaki H, Wahegaonkar A, Addosooki A. Fingertip replantation. J Hand Surg Am 2007;32(4);548-55. https://doi.org/10.1016/j.jhsa.2007.01.019

2. Rush MD, Grunert BK, Sanger JR, Dzwierzynski WW, Matloub HS. Psychological adjustment in children after traumatic disfiguring injuries: a 12 month follow up. Plast Reconst Surg 2000;106(7):1451-60. https://doi.org/10.1097/00006534-200012000-00001

3. Matsuzaki H, Yoshizu T, Maki Y, Tsubokawa N. Functional and cosmetic results of fingertip replantation: anastomosing only the digital artery. Ann Plast Surg 2004;53(4):353-9. https://doi.org/10.1097/01.sap.0000137136.09890.18

4. Patradul A, Ngarmukos C, Parkpian V. Distal digital replantations and revascularizations: 237 digits in 192 patients. J Hand Surg Br 1998;23(5):578-82. https://doi.org/10.1016/s0266-7681(98)80005-3

5. Foucher G, Norris RW. Distal and very distal digital replantations. Br J Plast Surg 1992;45(3):199-203. https://doi.org/10.1016/0007-1226(92)90076-a

6. Buntic RF, Brooks D. Standardized protocol for artery-only fingertip replantation. J Hand Surg Am 2010;35(9):1491-6. https://doi.org/10.1016/j.jhsa.2010.06.004

7. Erken HY, Takka S, Akmaz I. Artery-only fingertip replantations using a controlled nailbed bleeding protocol. J Hand Surg Am 2013;38(11):2173-9. https://doi.org/10.1016/j.jhsa.2013.08.110 
8. Yi-Chieh Chen, Fuan Chiang Chan, Chung-Chen Hsu, Yu-Te Lin, Chien-Tzung Chen, Chih-Hung Lin. Fingertip replantation without venous anastomosis. Ann Plast Surg 2013;70(3):284-8. https://doi.org/10.1097/SAP.0b013e3182321b81

9. Slattery P. Distal digital replantation using a solitary digital artery for arterial inflow and venous drainage. $J$ Hand Surg Am 1994;19(4):565-6. https://doi.org/10.1016/0363-5023(94)90257-7

10. Fukui A, Maeda M, Inada Y, Tamai S, Sempuku T. Arteriovenous shunt in digit replantation. J Hand Surg Am 1990;15(1):160-5. https://doi.org/10.1016/s0363-5023(09)91125-X

11. Kamei K, Sinokawa Y, Kishibe M. The venocutaneous fistula. A new technique for reducing venous congestion in replanted fingertips. Plast Reconstr Surg 1997;99(6):1771-4. PMID: 9145156

12. Tamai S. Twenty years' experience of limb replantation: Review of 293 upper extremity replants. J Hand Surg Am 1982;7(6):549-56. https://doi.org/10.1016/s0363-5023(82)80100-7

13. Ishikawa K, Ogawa Y, Soeda H, Yoshida Y. A new classification of the amputation level for the distal part of the finger. J Jpn Soc Microsurg 1990;3:54-62.

14. Hui-Fu Huang, Eng-Kean Yeong. Surgical treatment of distal digit amputation: Success in distal digit replantation is not dependent on venous anastomosis. Plast Reconstr Surg 2015;135(1):174-8.

https://doi.org/10.1097/PRS.0000000000000796 